\title{
Persistent eyelid swelling in a child: lest myiasis be forgot
}

\author{
Eda Karadag-Oncel • Ali Bulent Cengiz • \\ Buse Ozer-Bekmez
}

Received: 9 October 2013 / Accepted: 20 November 2013 /Published online: 3 December 2013

(C) Springer-Verlag Berlin Heidelberg 2013

\begin{abstract}
Myiasis involving the eyelid is uncommon. Herein, we present an 8-year-old boy who was diagnosed with preseptal cellulitis, yet a single larva was eventually extracted from his eyelid.
\end{abstract}

Keywords Myiasis $\cdot$ Eyelid swelling $\cdot$ Children

\section{Patient}

An 8-year-old boy from central Anatolia presented to the outpatient clinic with swelling in the upper eyelid of the left eye. Physical examination revealed the presence of solid edema of the left upper lid, which was isolated to regions anterior to the orbital septum. On review of past medical history, it was discovered that for the previous 6 months, the patient had complained of migratory dermal swellings, roughly $2-4 \mathrm{~cm}$ in size, which reappeared every 15-45 days, each lesion persisting for 1-3 days at a time. With a preliminary diagnosis of preseptal cellulitis, the patient was started on a course of amoxicillin clavulanic acid combined with an antihistaminic since angioedema could not be ruled out. Two days later, a single larva, which had emerged spontaneously from the pore in the upper eyelid followed by immediate partial resolution of the swelling (Fig. 1) was retrieved and sent for parasitological analysis. Our parasitology department confirmed a diagnosis of myiasis,

E. Karadag-Oncel $(\bowtie) \cdot$ A. B. Cengiz $\cdot$ B. Ozer-Bekmez Department of Pediatrics, Division of Pediatric Infectious Diseases, Faculty of Medicine, Hacettepe University, Sihhiye 06100, Ankara, Turkey

e-mail: dredakaradag@gmail.com but the particular species could not be identified due to a technical issue. The patient's ocular findings eventually returned to normal.

Myiasis is the invasion of a host's tissues by the larval stage (maggot) of nonbiting flies. More than 50 fly species have been identified as a cause of myiasis in humans. Previously reported sites of infestation include the skin, eye, ear, nasopharynx, genitourinary tract, intestine, and open wounds. Although rare, myiasis should be considered in the differential diagnosis of eyelid swelling in children presenting with periorbital cellulitis, and the presence of any breach or pore in the skin warrants careful examination.

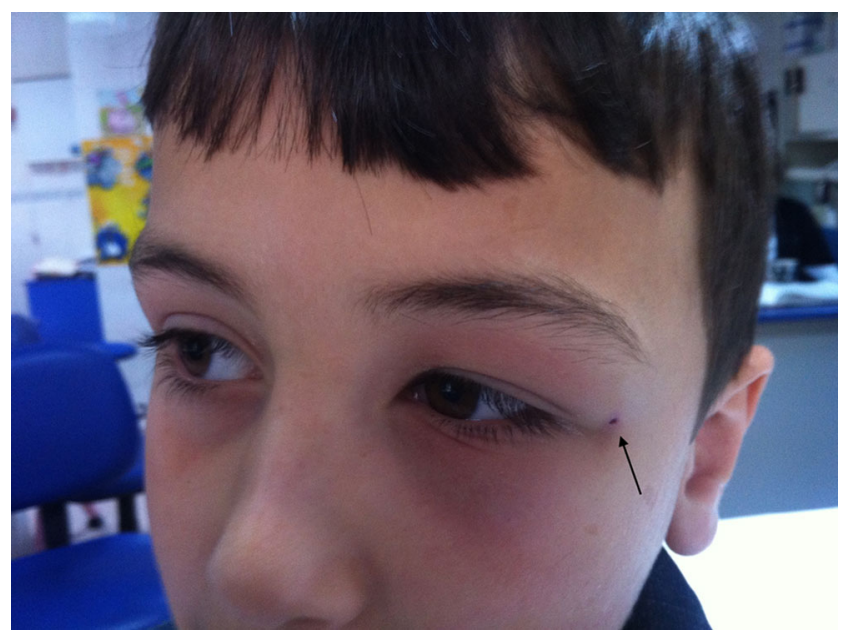

Fig. 1 Photograph showing partial resolution of eyelid swelling with the presence of a respiratory pore (black arrow)

Conflict of interest None 